\title{
A Formal, Bayesian Approach for Uncertainty Analysis of a Watershed Model
}

\author{
Misgana K. Muleta \\ Department of Civil and Environmental Engineering, California Polytechnic State University, San Luis Obispo, California 93407; email: \\ mmuleta@calpoly.edu.
}

\begin{abstract}
Uncertainty analysis (UA) has received substantial attention in water resources during the last decade. Bayesian approaches are often preferred for UA. This study describes a formal Bayesian approach for the assessment of parameter uncertainty and predictive uncertainty using a spatially distributed hydrologic model and will demonstrate its application using data from a well monitored experimental watershed. A Markov-Chain Monte Carlo (MCMC) scheme has been used to sample posterior parameter distributions. A formal, flexible likelihood function that explicitly accounts for heteroscedasticity, temporal correlation and non-normality of simulation residuals has been used to describe closeness of the simulated and observed streamflow. Performance of the formal likelihood function will be compared to that of simple least squares with regard to generating accurate predictive uncertainty estimates at multiple streamflow gaging stations available in the experimental watershed. Limitation of the SLS assumptions with regard to the structure of model residuals will be illustrated and capability of the formal likelihood function to address these assumptions will be scrutinized. Finally, the maximum likelihood solutions identified by the uncertainty analysis method will be compared to the optimal solutions determined using a single objective optimization exercise to test effectiveness of the uncertainty analysis method to also identify the optimal solutions sought during model calibration.
\end{abstract}

\section{Introduction}

Recognizing the need to quantify the uncertainty associated with model simulations and reduce potential adverse impacts of making decisions based on unreliable model predictions, uncertainty analysis (UA) has received significant attention in hydrology (Beven and Freer, 2001; Muleta and Nicklow, 2005; Kavestki et al., 2006; Montanari et al., 2009; Schoups and Vrugt, 2010). The inability to accurately measure and/or account for spatial and temporal variability of model forcings, measurement errors associated with the observations used to test and calibrate models, inadequacy of the simulation model to accurately represent hydrologic processes of a watershed, and the uncertainty emerging from the challenge to identify reasonable values of model parameters are believed to be the major sources of uncertainty in hydrologic modeling (Moradkhani and Sorooshian, 2008; Gotzinger and Bardossy, 2008 ). While some studies have attempted to decompose the total uncertainty to quantify contribution of the individual uncertainty sources (Gotzinger and Bardossy, 2008), the common practice is to quantify the total predictive uncertainty by aggregating contributions from all sources (Renard et al, 2010; Schoups and Vrugt, 2010).

The Generalized Likelihood Uncertainty Estimation (GLUE) approach of Beven and Binley (1992) has been the most commonly used UA technique in water resources during the last decade. Recently, several authors have questioned statistical validity of the GLUE approach (Mantovan and Todini, 2006; Stedinger et al., 2008; Vrugt et al., 2008). To address concerns against informal techniques such as GLUE approach, formal UA methods that employ statistically valid likelihood measures within the Bayesian framework are emerging (Schoups and Vrugt, 2010). The Bayesian UA framework determines posterior probability density function 
(pdf) of model parameters as well as total predictive uncertainty, e.g. through Monte Carlo simulations. Because of their relative computational efficiency, Markov-Chain Monte Carlo (MCMC) schemes have been preferred to the standard Monte Carlo simulations that rely on random sampling for UA applications. The Metropolis Hasting algorithm (MH) (Hastings, 1970) is the foundation of the MCMC sampling schemes. MH schemes, however, could be very inefficient depending on the initialization of the proposal distribution (i.e., the variance of the distribution) being either too wide or too narrow to efficiently converge to a stable posterior pdf (Vrugt et al., 2008). Several approaches have been introduced to improve efficiency of the MH algorithm. A recently developed efficient MCMC scheme known as $\operatorname{DREAM}_{(\mathrm{ZS})}\left(\operatorname{Schoups}_{\mathrm{B}} \mathrm{and}\right.$ Vrugt, 2010) has been used in this study.

Efficient and robust sampling method is a prerequisite to successfully adopting Bayesian analysis. Yet, the reliability of the sampled posterior distribution, and corresponding estimates of parameter and model predictive uncertainty essentially relies on the formulation of the likelihood function used to summarize the mismatch between model predictions and data. There is a general consensus that with proper assumptions, formal likelihood measures (as opposed to the informal likelihood functions), would produce statistically valid and more realistic confidence intervals (Mantovan and Todini, 2006; Vrugt et al., 2008; Stedinger et al., 2008; Schoups and Vrugt, 2010). However, the formal UA applications commonly reported in water resources modeling make unrealistic assumptions regarding structure of the residuals between model simulations and the observed watershed behavior. These assumptions include 1) the residuals are independence (i.e., no correlation between errors of successive time steps), 2) the residuals follow Gaussian distribution, 3) the results are homoscedastic (i.e., error variances do not depend on magnitude of the streamflow). Addressing all these unjustifiable assumptions, a generalized formal likelihood (GL) function has been recently described by Schoups and Vrugt (2010), and has been used for this study.

Most UA applications in hydrology that use MCMC techniques within the Bayesian framework demonstrated their findings using lumped-conceptual models (Vrugt et al., 2008, Vrugt et al., 2009; Kuczera et al., 2010; Schoups and Vrugt, 2010). Lumped models cannot explicitly account for spatial variability of watershed response; they have a relatively low number of model parameters; they are computationally less demanding; and they are commonly used for rainfall-runoff (R-R) modeling applications only as they lack the capability to simulate other important watershed fluxes such as water quality variables. Using lumped models, capability of a UA technique cannot be adequately examined with regard to generating accurate predictive uncertainty at sites other than the calibration location (e.g., at internal gages not used for calibration). Application to watershed models that have large number of parameters and are computationally more demanding would further illustrate practicality of the UA techniques.

By applying DREAM $(\mathrm{ZS})$ and the GL function to a spatially distributed and semiphysically based watershed model, this study examines robustness of the UA technique for water resources models that are more often used in many decision making applications. The watershed model selected for this study is the Soil and Water Assessment Tool (SWAT) (Arnold et al., 1999), and a relatively data rich experimental watershed located in Georgia, United States, has been used as illustrative case study. The application explores capability of the UA technique to generate accurate predictive confidence intervals at several internal sites in the demonstration watershed. Another major objective of this study is to investigate seasonal variation of SWAT parameters based on the parameter uncertainty determined using $\operatorname{DREAM}_{(\mathrm{ZS})}$ and GL. A previous study (Muleta, 2011a) has demonstrated seasonal variation of the optimal model 
parameters identified using a single objective calibration exercise for the watershed used in this study. The seasonal sensitivity will be verified in this study.

\section{The DREAM(ZS) Algorithm}

Improving effectiveness and efficiency of the MCMC schemes has been one of the ingredients of the uncertainty analysis research. In this regard, various publications have shown that multichain sampling approaches that use different trajectories in parallel to search the parameter space in pursuit of the posterior distribution have desirable properties. The use of multiple chains not only protects against premature convergence, but also opens up a wide arsenal of statistical measures to assess when a limiting posterior distribution has been found (Gelman and Rubin, 1992). Moreover, the solutions visited with the different chains can be used to continuously update the scale and orientation of the proposal distribution. Examples of multi-chain methods include the Shuffled Complex Evolution Metropolis (SCEM-UA) algorithm and DiffeRential Evolution Adaptive Metropolis (DREAM) scheme (Vrugt et al., 2008).

The number of parallel chains, $N$, required by standard DREAM such as the Differential Evolution Markov chain (DE-MC) method of ter Braak (2006) is at least $N=d / 2$ to $d$ where $d$ is the dimensionality of the problem which refers to the number of parameters to be estimated. Running many parallel chains is a potential source of inefficiency as each individual chain requires burn-in to travel to the posterior distribution. Lowering the number of chains would improve applicability of DREAM for computationally demanding posterior exploration problems such the spatially distributed modeling application considered in this paper. One approach that enables using a smaller $N$ is to improve information sharing among the chains by generating candidate points from past states of the different chains

These findings inspired Vrugt et al. (2011) to create $\operatorname{DREAM}_{(\mathrm{ZS})}$ that capitalizes on the advantages of DREAM for posterior exploration but generates candidate points in each individual Markov chain by sampling from an archive of past states. This has several practical and theoretical advantages. Most importantly, only a few parallel chains $(N=3$ to 5) are required for posterior sampling. This reduces burn-in, particularly for problems involving many parameters (large $d$ ), thereby increasing sampling efficiency (Vrugt et al., 2011). In DREAM(Zs), the states of the chains are periodically stored in an archive using a simple thinning rule. The size of this matrix steadily increases during sampling, but the relative growth decreases linearly with generation number. This diminishing adaptation of the transition kernel ensures convergence of the individual chains to the posterior distribution. To increase the diversity of the proposals, $\operatorname{DREAM}_{(Z S)}$ additionally includes a snooker updater with adaptive step size. The snooker axis runs through the states of two different chains, and the orientation of this jump is different from the parallel direction update utilized in DREAM. The algorithmic implementation of the snooker update within the context of DE-MC is described in ter Braak and Vrugt (2008). This study explores robustness of $\operatorname{DREAM}_{(\mathrm{ZS})}$ for spatially distributed modeling.

\section{The Watershed Simulation Model}

Soil and Water Assessment Tool (Arnold et.al., 1999), the simulation model used for this study, is one of the most widely-used watershed simulation models in use today (Gassman et al., 2007). SWAT is a physically-based and spatially-distributed model that uses information regarding climate, topography, soil properties, land cover, and human activities such as land management practices to simulate numerous physical processes including surface runoff, groundwater flow, 
streamflow, sediment concentration, pesticides, nutrients such as nitrogen and phosphorous, pathogens and bacteria. Spatially, the model subdivides a watershed in to subwatersheds and, potentially, further partitions subwatersheds into hydrologic response units (HRUs) based on land cover, soil, and overland slope diversity in the subwatersheds. Major hydrologic processes modeled by SWAT include snowpack and snow melt, surface runoff, potential evapotranspiration, estimated by Penman-Monteith, Hargreaves or Priestley method; percolation, simulated by a combination of a layered routing technique with a crack flow model; lateral subsurface flow or interflow, simulated by a kinematic storage model; and ground water flow. SWAT operates within ESRI's ArcGIS platform greatly simplifying the preparation of model inputs and visualization of outputs. In this study, SWAT version 2005 (Neitsch et al., 2005) has been used to solve the governing watershed processes, and to determine streamflow outputs at desired locations throughout the demonstration watershed. The reader is referred to Neitsch et al. (2005) for technical details of the processes modeled by SWAT.

\section{The Study Watershed and Data}

Headwaters of the Little River Experimental Watershed (LREW), one of the USDA-ARS's experimental watersheds, located in Geogria, United States, will be used as a case study. The LREW has been selected because it is heavily gaged for rainfall as well as streamflow (Bosch et al, 2006), and because data are readily accessible online (ftp://www.tiftonars.org/) from the Southeast Watershed Research Laboratory (SEWRL). The watershed consists primarily of lowgradient streams and is located mainly on sandy soils underlain by limestones that form locally confined aquifers. Land use within the watershed consists of about $31 \%$ row crop agriculture, $10 \%$ pasture, $50 \%$ forest, and $7 \%$ urban area (Bosch et al. 2006).

Only the upper $116 \mathrm{~km}^{2}$ of the LREW has been used for this study to minimize computational demand of the model, and because the headwater subwatersheds have denser streamflow and rainfall gages. Twelve precipitation gages and five streamflow gages with longterm daily data (i.e., 1967-2006) are available for the headwaters of the SEWRL. Daily minimum and daily maximum temperature data for a station near the watershed has been obtained from the U.S. Historical Climatology Network (http://cdiac.ornl.gov/epubs/ndp/ushcn/ushcn.html) as the air temperature data available from SEWRL starts only from 2004. The geographic data used to setup the SWAT model including topography, land use, stream networks, and rainfall and streamflow gauging locations have been obtained from the SEWRL. SSURGO soil map has been obtained from the Natural Resources Conservation Service (NRCS) soil data mart (http://soildatamart.nrcs.usda.gov/). SWATioTools (Sheshukov et al., 2009), an ArcMap GIS extension tool that converts SSURGO soil maps into a format readable by ArcSWAT (Winchell et al., 2008) has been used to preprocess the SSURGO soil map. The land cover image used for the study area was for year 2003, and was also preprocessed to synchronize the land cover names used in the original map with SWAT's land cover types. After the climate, streamflow, land use, and soil data were preprocessed, the $116 \mathrm{~km}^{2}$ study watershed was delineated and subdivided into 37 subbasins and 71 HRUs using ArcSWAT.

\section{Methodology}

Objectives of this study are to examine robustness of $\operatorname{DREAM}_{(\mathrm{ZS})}$ using a spatially distributed watershed model and to examine relative advantages of the GL function over the commonly used SLS approach. Seasonal sensitivity of SWAT parameters will also be further investigated using 
the UA technique pursued here. The subsequent paragraphs describe the methodologies used in this work to (1) identify the SWAT parameters included in the analysis and define prior pdfs and bounds for the parameters; (2) determine posterior pdfs for the parameters; (3) compute streamflow predictive uncertainty at multiple gaging sites.

\section{Parameter Selection}

As a spatially distributed hydrologic model, the number of SWAT parameters may become very large depending on the number of subwatersheds and HRUs in the watershed. Streamflow and other watershed responses, however, may not be equally sensitive to all model parameters. In an attempt to identify SWAT parameters that are most influential in describing streamflow for the watershed used in this study (i.e., LREW), a detailed sensitivity analysis (SA) based on Sobol' (Sobol', 1993) has been previously performed (Muleta, 2011a). Sobol's method is a variancebased SA approach that determines first-order sensitivity as well as total sensitivity indices for each parameter accounting for higher order interaction effects among the parameters. This previous study identified twelve SWAT parameters that play a crucial role in simulating streamflow for the watershed. Optimal values for these parameters were previously identified using single objective calibration (Muleta, 2011a; Muleta, 2011b). Although nine different objective functions were examined for the single objective optimization performed by Muleta (2011b), the results obtained using the root mean square errors (RMSE) has been used for this study to be consistent with the SLS approach.

Seasonal model evaluation was used in these previous studies, i.e., model parameters were allowed to vary between dry and wet seasons during model calibration. In the traditional model evaluation approach, parameters are assumed to be constant and are assigned the same value during low flow seasons as well as high flow seasons. The study by Muleta (2011a) showed significant improvement in model performance when this dynamic model evaluation approach is pursued compared to the commonly used constant parameter approach. Dry and wet seasons were defined using monthly runoff coefficients determined from 39 years (i.e., 19682006) of rainfall and runoff data for the watershed (Muleta, 2011a). Months with runoff coefficient greater than 0.1 (i.e., January to April) were considered wet season, and months with runoff coefficient less than 0.1 (i.e., June to November) were considered dry season. December and May were considered transition months where parameters values would linearly vary from the dry season values to the wet season values and vice versa.

Nine of the twelve most influential streamflow parameters were allowed to vary from wet season to dry season making the total number of model parameters to be calibrated twenty-one. In the current study, in an attempt to reduce computational demand, only the six most sensitive SWAT's streamflow parameters were considered for the uncertainty analysis. Five of the six parameters were allowed to assume different values for the dry and wet seasons thus increasing the total number of parameters to eleven. The other ten influential SWAT parameters were assigned the optimal values obtained by the previous calibration efforts (Muleta, 2011a; Muleta, 2011b).

Prior pdfs for all eleven parameters considered for the uncertainty analysis were assumed to be uniform distribution as done in Muleta and Nicklow (2005), and the lower and upper bounds recommended in Neitsch et al. (2005) were used for most parameters. Some of these model parameters (e.g., NRCS's curve number, CN2) vary from HRU to HRU, from subbbasin to subbasin, or from reach to reach depending on soil, land cover, slope and/or other watershed characteristics. SWAT determines CN2 values for the HRUs in a subwatershed based on land 
cover and soil type of the HRU and calculates runoff and other responses at the scale of HRU based on the characteristics of the HRU (e.g., its CN2 value). During the UA, spatially varying parameters were altered by multiplying their baseline values with multipliers, or alternatively by adding the sampled DREAM $(\mathrm{ZS})$ values to their respective baseline values. This approach preserves the a-priori specified spatial variability, and assumes that this variability of the baseline values determined by ArcSWAT (Winchell et al., 2008) is representative of the watershed characteristics but might have been over/under estimated consistently across the watershed. An alternative approach is to allow the adjustments (multiplicative or additive) to vary for each spatial unit (e.g., unique $C N 2$ adjustments for each of the 71 HRUs in the watershed). This approach, however, significantly increases the dimensionality of the parameter estimation problem depending on the number of spatial units in the watershed.

\section{Determining Posterior PDF for the Parameters}

Posterior distribution of the eleven SWAT parameters is estimated with DREAM(ZS) using the SLS and GL function. In the case of GL, four additional error model parameters were considered to explicitly account for heteroscedasticity, non-normality, and autocorrelation of the model residuals. A total of 50,000 SWAT simulations were used to sample posterior distribution of the model parameters and/or additional likelihood function error variables. Four years of daily streamflow data measured at the outlet of the watershed was used as calibration data. A one-year spin-up period was used to reduce sensitivity to state-value initialization (antecedent conditions), and the remaining three years (years 2000 - 2002) were used for posterior estimation.

Convergence of $\operatorname{DREAM}_{(\mathrm{ZS})}$ to a stable posterior pdf was monitored using the $\hat{R}$ statistic of Gelman and Rubin (1992). This diagnostic compares the between and within variance of the difference chains. Convergence is declared when $\hat{R}_{j} \leq 1.2$ for all $j=1, \ldots, d$, where $d$ represents the number of parameters. The last 10,000 simulations that meet the convergence criteria were extracted and parameter uncertainty (e.g., 95\% confidence interval)) was determined and reported for each individual parameter. To examine seasonal influences on the values of the parameters, we derive and compare posterior parameter distributions for the wet and dry season.

\section{Predictive Uncertainty Estimation and Testing}

Once the posterior distribution of the model parameters is known, streamflow predictive uncertainty can be estimated by propagating the different samples of the posterior distribution through the SWAT model, and reporting the respective $95 \%$ prediction uncertainty ranges. This prediction interval, however, represents parameter uncertainty only, without recourse to considering other sources of error, including model structural, forcing data, and calibration data uncertainty. Total predictive uncertainty has been determined using the approach described in Schoups and Vrugt (2010). Using parameter sets that were sampled from their respective posterior pdfs, five thousand SWAT simulations were performed to determine daily streamflow at five gages in the watershed from 2000-2006. Total predictive uncertainty was determined by adding SWAT's streamflow predictions to the independent error samples generated using the algorithm described in Schoups and Vrugt (2010).

Performance of the uncertainty analysis approach was verified using a traditional split sampling approach (i.e., the 2003- 2006 data at the calibration site, was used to verify the prediction intervals) as well as by analyzing the capability of the model to generate accurate predictive uncertainty intervals at other (internal) gages not used for calibration for the 
calibration as well as evaluation periods (i.e., 2000-2006). Both time series plot and quantilequantile plots, also called QQ graphs, were used to graphically depict the correspondence between the modeled and observed streamflow. The relative performance of the SLS and the GL approaches was also analyzed by comparing their estimates of parameter and prediction uncertainty. Finally, the ML parameter values for both likelihood functions are benchmarked against previous calibration using the Dynamically Dimensioned Search (DDS) method (Tolson and Shoemaker, 2007), and are also compared in terms of their ability to fit different parts of the hydrograph. The solution methodology and results will be detailed during the presentation at the conference.

\section{References}

Arnold, J.G., Williams, J.R., Srinivasan, R., King, K.W.(1999). "SWAT: Soil and Water Assessment Tool.” U.S. Dept. of Agriculture, Agricultural Research Service, Temple, TX.

Beven, K. J., and A. M. Binley (1992). The future of distributed models: Model calibration and uncertainty prediction, Hydrol. Processes, 6, 279-298, doi:10.1002/hyp.3360060305.

Beven, K., and J. Freer (2001), Equifinality, data assimilation, and uncertainty estimation in mechanistic modeling of complex environmental systems using the GLUE methodology, J. Hydrol., 249, 11-29.

Bosch, D.D., Sullivan, D.G., Sheridan, J. (2006). "Hydrologic impacts of land-use changes in coastal plain watersheds." Transactions of the ASABE, 49(3), 423-432.

Gassman, P.W., Reyes, M.R., Green, C.H., and Arnold, J.G.(2007). "The Soil and Water Assessment Tool: Historical Development, Applications, and Future Research Directions." Transactions of the ASABE, 50(4), 1211-1250.

Gelman, A. and D. B., Rubin (1992), Inference from Iterative Simulation Using Multiple Sequences. Statistical Science, 7, 457-472.

Gotzinger, J., and A. Bardossy (2008), Generic error model for calibration and uncertainty estimation of hydrological models, Water Resour. Res., 44, W00B07, doi:10.1029/2007WR006691.

Hastings, H. (1970). Monte Carlo sampling methods using Markov chains and their applications, Biometrika, 57, 97-109

Kuczera, G., D. Kavetski, B. Renard, and M. Thyer (2010), A limited memory acceleration strategy for MCMC sampling in hierarchical Bayesian calibration of hydrological models, Water. Resour. Res., 46, W07602, doi:10.1029/2009WR008985

Mantovan, P., and E. Todini (2006), Hydrological forecasting uncertainty assessment: Incoherence of the GLUE methodology, J. Hydrol., 330, 368- 381.

Montanari, A., C. A. Shoemaker, and N. van de Giesen (2009), Introduction to special section on Uncertainty Assessment in Surface and Subsurface Hydrology: An overview of issues and challenges, Water Resour. Res., 45, W00B00, doi:10.1029/2009WR008471.

Moradkhani, H. and Sorooshian, S.: "General Review of Rainfall-Runoff Modeling: Model Calibration, Data Assimilation, and Uncertainty Analysis", Book Chapter in Hydrological Modeling and Water Cycle, Coupling of the Atmospheric and Hydrological Models, Springer, Water Science and Technology Library, Volume 63, pp 1-23, 2008.

Muleta, M.K., 2011a. Improving Model Performance Using Season Based Evaluation. J. Hydrologic Engrg., ASCE, in press. 
Muleta, M.K., 2011b. Sensitivity of Model Performance to the Efficiency Criteria Used as Objective Function during Automatic Calibrations. J. Hydrologic Engrg., ASCE, in review.

Muleta, M.K., Nicklow, J.W., 2005. Sensitivity and uncertainty analysis coupled with automatic calibration for a distributed watershed model. J. Hydrol., 306, 1-19.

Neitsch, S.L., Arnold, J.G., Kiniry, J.R., Williams, J.R.(2005). "Soil and Water Assessment Tool-Version 2005-Theoretical Documentation”, Temple, Texas, USA.

Renard, B., D. Kavetski, G. Kuczera, M. Thyer, and S. W. Franks (2010), Understanding predictive uncertainty in hydrologic modeling: The challenge of identifying input and structural errors, Water Resour. Res., 46, W05521, doi:10.1029/2009WR008328.

Schoups, G., and J. A. Vrugt (2010), A formal likelihood function for parameter and predictive inference of hydrologic models with correlated, heteroscedastic, and non - Gaussian errors, Water Resour. Res., 46, W10531, doi:10.1029/2009WR008933.

Sheshukov, A., Daggupati, P., Lee, M. C., Douglas-Mankin, K.(2009). "ArcMap Tool for Preprocessing SSURGO Soil Database for ArcSWAT." Proceedings of the 5th International SWAT Conference, Boulder, CO., Aug. 5-7.

Sobol', I.M. (1993). "Sensitivity estimates for non-linear mathematical models", Math. Modeling Comput. Experiment, 1(4), 407-414.

Stedinger, J. R., R. M. Vogel, S. U. Lee, and R. Batchelder (2008), Appraisal of the generalized likelihood uncertainty estimation (GLUE) method, Water Resour. Res., 44, W00B06, doi:10.1029/2008WR006822.

ter Braak, C. J. F. (2006), A Markov Chain Monte Carlo v860 ersion of the genetic algorithm differential evolution: Easy Bayesian computing for real parameter space, Stat. Comput., 16 (3), 239249, doi:10.1007/s11222-006-8769-1.

Tolson, B. A., Shoemaker, C. A.(2007). "Dynamically dimensioned search algorithm for computationally efficient watershed model calibration." Water Resour. Res., 43, W01413, doi:10.1029/2005WR004723.

Vrugt, J.A., C.J.F., ter Braak, C. G.H. Diks, J. Rings and G. Schoups, 2011, A Particle DREAM Filter for Posterior Tracking of Hydrologic Model Parameters and States, Water Resour. Res., Under Review.

Vrugt, J. A., C. J. F. ter Braak, C. G. H. Diks, D. Higdon, B. A. Robinson, and J. M. Hyman (2009), Accelerating Markov chain Monte Carlo simulation by differential evo-lution with self-adaptive randomized subspace sampling, Int. J. Nonlinear Sci. Numer. Simul., 10(3), 273-290.

Vrugt, J. A., C. J. F. ter Braak, H. V. Gupta, and B. A. Robinson (2008), Equifinality of formal (DREAM) and informal (GLUE) Bayesian approaches to hydrologic modeling, Stoch. Env. Res. Risk A., 23, 1059-1060, doi:10.1007/s00477-008-0274-y.

Winchell, M., Srinivasan, R., Di Luzio, M., Arnold, J. (2008). "ArcSWAT 2.1 Interface for SWAT 2005: User's Guide”, Blackland Research Center, Temple, Texas. 